\title{
Implementation of Stepped Frequency Modulation Pulse Compression on NI Suite
}

\author{
D.Tamilarasi, P. Pavithra, P. Ramesh
}

\begin{abstract}
In Radars and Sonar, the pulse compression technique is used continuously to increase the range resolution, range detection and the signal-to noise ratio (SNR). This can be achieved by modulating the transmitted pulse and then correlating it to the received pulse with the transmitted signal. This transforms short pulse into long pulse and is used to increase long pulse bandwidth by some form of modulation such as linear frequency modulation (LFM), so that Range Resolution is not compromised. The proposed Stepped Frequency Modulation (SFM) is a common Pulse Compression Method, which is useful to increase the Radar Range Resolution without losing the capability of target detection. Step Frequency Continuous Wave (SFCW) is also one of the techniques used in the Pulse Compression Technique, where the return echo step is used to determine range and is used for different purposes. This type of setup is widely used in RADAR design and testing. This Paper proposes the implementation of various Modulation techniques such as LFM, SFM and SFCW for proposed Stepped frequency Modulation using NI suite hardware PXI system which has a configurable FPGA and RF front end to generate custom waveform in wide range of frequencies with bandwidth up to $1 \mathrm{GHz}$ design and testing.
\end{abstract}

Keywords: Radar, Linear Frequency Modulation, Pulse compression, Step frequency Continuous Wave, NI suite.

\section{INTRODUCTION}

RADAR is a system used for detecting an object in the free space, ground and undersea. It sends pulses and the object's echo is used to determine the detected object's location, distance, width, etc. PULSED RADAR is widely used in RADAR and works on the principle of Doppler Effect. Range Resolution is a Radar advantage in detecting and distinguishing between two closely spaced targets. Airborne Radar usually operates in X-band. If the frequency of the radar reduces it does not interfere with the particles of atmosphere and hence range for detection of target reduces. If frequency of the radar increases then it interferes with the

Revised Manuscript Received on December 30, 2019.

* Correspondence Author

D.Tamilarasi*, PhD, Associate Professor, Department of ECE, MVJ college of Engineering, Bengaluru, India. E-mail: dr.tamilarasi@mvjce.edu.in

P.Pavithra, Assistant Professor, Department of ECE,MVJ college of Engineering, Bengaluru, India. E-mail: pavithra..p@mvjce.edu.in

P.Ramesh PhD, Associate Professor, Department of EEE ,CMR Institute of Technology, Bengaluru, India. E-mail: ramesh8889@gmail.com

(C) The Authors. Published by Blue Eyes Intelligence Engineering and Sciences Publication (BEIESP). This is an open access article under the CC BY-NC-ND license (http://creativecommons.org/licenses/by-nc-nd/4.0/) particles of atmosphere, but the range for detection of target increases. Hence radio waves with low frequency resist the particles. But, radio waves with high frequency offer less resistance to the particles present in atmosphere and hence allow these particles to interfere with radio waves.

Airborne Radars use pulse repetition frequencies (PRF) from few hundred Hertz to several hundred kilo Hertz to estimate target unambiguous. The frequency of pulse repetition (PRF) is the number of pulses of a repeating signal measured in pulses per second in a specific time unit. PRF determines the extent to which the range and Doppler frequencies will be unambiguous. The three basic categories of PRF are low, medium, and high PRF. [1] A technique in which a combination of the incoming signal and the one-bit shifted version of the incoming signal is applied to the amplitude weighting. This technique produces better lobe ratio of peak side (PSL) and integrated side lobe ratio (ISL) than all other traditional side lobe reduction techniques. Like other application-specific integrated circuits (ASIC), the design has distinct advantages.

For example, the FPGA provides full reconfiguration in milli-seconds and allows for a complete single chip solution. In addition to overcoming the peak-power limitations, pulse compression radars have an EMC (Electromagnetic Compatibility) advantage in making them more tolerant to interference with each other. This is achieved by allowing the pulse-compression radar operating within a given band to have its own characteristic modulation and its own matched filter [2]. Dodda H.V.S. Rami Reddy has achieved experimental results which confirm that the complementary codes have best characteristic ACF and AF in comparison with all the rest of the codes, reducing the noise levels to zero [3]. Anuja D. Sarate explored the merits and demerits of various LFM, Biphase, and Polyphase pulse compression techniques. Considering the codes are known. In my proposed model, the noise level is subsequenty suppressed and an efficient pulse compression is achieved [4].

\section{PULSE COMPRESSION TECHNIQUES}

(i)Pulse compression with Linear FM waveform Pulse compression is very common in modern radar using li near frequency modulation techniques. The linear frequency modulation is used to address two small targets with very lit tle spacing between them, which are located at long range. LFM is the most common radar system and is listed as both analog and digital. Over time, the frequency increases linearly, so it is called linear modulation of frequency. 


\section{Implementation of Stepped Frequency Modulation Pulse Compression on NI Suite}

To achieve broad operating bandwidth, Linear Frequency Modulation is used. The LFM waveform simulation is conducted for different specifications. The LFM signal is generated and correlated with the same complex conjugate, simulating the corresponding filter response. In any case, the length of the pulse is $20 \mu \mathrm{s}$. Simulations are performed for the variation of bandwidth from $100 \mathrm{MHz}$ to $250 \mathrm{MHz}$. Pulse compression is very popular in modern radar using linear frequency modulation techniques. The linear frequency modulation is used to solve two small targets with very small separation between them, which are located at long range. Due to good range resolution and sensitivity to Doppler, LFM is the most common radar waveform. Through analog and digital techniques, LFM waveform generation schemes are named. Over time, the frequency increases linearly, so it is called linear modulation of frequency.

To achieve broad operating bandwidth, Linear Frequency Modulation is used. Figure 1 shows that the LFM signal is a type of signal that varies the frequency of the transmitted signal over the TP pulse length. Changing the frequency from low to high is called "up-chirp" or similar upsweep, it is called "down-chirp" to change the frequency from high to low. The LFM waveform simulation is conducted for different specifications. The LFM signal is generated and correlated with the same complex conjugate, simulating the corresponding filter response. In any case, the duration of the pulse is $20 \mu \mathrm{s}$. Simulations are conducted for the difference of bandwidth from $100 \mathrm{MHz}$ to $250 \mathrm{MHz}$. You may modify those characteristics of the waveform to create a linear FM pulse waveform, including sample rate, single pulse length, pulse repeat frequency, sweep range, sweep direction (up or down), corresponding to and and decreasing instantaneous frequency .

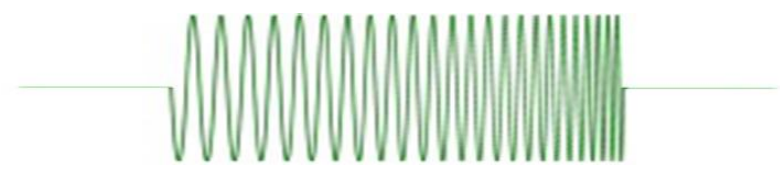

Figure 1 A Linear frequency modulated pulse

S will which the pulse waveform's amplitude

Modulation. The shell may be Gaussian or rectangular and it is expressed using equation 1 and 2.

\section{(ii)Equation of LFM}

$Y=\cos \left(2\left(f t+(k / 2) t^{2}\right)+j \sin \left(2\left(f t+(k / 2) t^{2}\right)\right) \ldots \ldots \ldots \ldots(1)\right.$

Where,

$f=$ frequency $(\mathrm{Hz})$

$$
k=\text { constant }=\frac{f 1-f 0}{2 \times \tau} \ldots \ldots \ldots(2)
$$

t=time period (sec) $\tau=$ pulse width

\section{(iii)Proposed Stepped Frequency Modulation}

Radar technology is now rapidly developing, with Step Freq uency Continuous Wave (SFCW) ,which determines the modulation of amplitude of the pulse waveform. This is also one of the methods used in the Pulse Compression Method, using the returning echo step in this method to evaluate distance and use it for different purposes. Stepped-frequency $\mathrm{CW}$ radar is basically a discrete version of FMCW that measures the frequency domain channel with $\mathrm{N}$ equally spaced in frequency $\mathrm{CW}$ measurements. At interfaces between materials with different dielectric constants, major radar reflection occurs. This results in reflections that the receiving antenna hears. For example, we obtain radar data sampled in the frequency domain using a hand held CW-SF radar device. The data consists of the values of I (in phase) and $\mathrm{Q}$ (quadrature) corresponding to the real and imaginary portions of the time-varying radar signal of a discrete Fourier transform (DFT). The SFCW in which it consists of frequency signal consisting of several steps continuously at a given time, as the name implies $\mathrm{CW}$ radar continuously broadcast radar waveform. Discrete Fourier Transform (DCT) is the most fundamental part of SFCW as the frequency is transmitted discreetly.The SFC wave analyzing and designing requires some important parameters those are: Pulse Repetition Frequency (PRF), numpulse. Pulse width (PW), some Matlab inbuilt commands and variables to develop SFCW code to analyze the wave forms and its mathematical equation is represented using equation.3.

\section{(iv) Equation for Proposed Step Frequency CW}

$$
\begin{aligned}
& Y=\cos \left(2\left(f_{0}+(n \Delta f) t\right)+j \sin \left(2\left(f_{o}+(n \Delta f) t\right) \ldots \ldots\right.\right. \\
& \text { Where, } \\
& \qquad \begin{array}{r}
f_{0}=\text { Initial frequency } \\
\Delta f=\text { constant frequency step change } \\
\mathrm{n}=\text { Number of pulses } \\
\mathrm{t}=\text { time period }
\end{array}
\end{aligned}
$$

\section{(v)Pulse compression with Stepped FM waveform}

The Stepped Frequency Waves are also a popular pulse compression technique, like the Linear Frequency Modulation (LFM) wave. Increasing the Radar Range Resolution without losing the capability of target detection in this technique is beneficial. This technique is particularly suitable for obtaining high-range resolution in a radar system that has limited instant bandwidth but a large tuneable bandwidth and allows the transmission of waveforms with extremely wide overall bandwidth without the use of expensive hardware necessary to support wide instantaneous bandwidth. "Stepped LFM is the LFM wave in which frequency increased from pulse to pulse in steps with constant frequency step (Delta ' $f$ ')" as shown in Figure2and its corresponding time domain and frequency response of LFM is given as shown in Figure 3 and 4. 
The Stepped LFM wave splits the full band width into a series of $\mathrm{N}$ pulses of pulse width(tow), duration (Tr) and each pulse has same band width, a large band width is achieved and its mathematically denoted using mathematical equation given in equation 4.In this technique, constant steps (delta f) are generated between the centre frequencies of consecutive pulses. Initial pulse has a frequency of ' 0 ' and is a DC pulse with the numpluses property set to 1 , the frequency of the narrowband pulse increases by the frequency phase property value every time we use step. The waveform is generated after applying FFT processing to the received frequency samples and modulation occurs in pulses instead of within the pulses. Scope resolution is the resolution of 2 small targets positioned on the long range with very little distance between them on the same bearing, high-range target resolution measurements provide more data about the target and provide more accurate target measurements. Some important parameters used to define and design the Stepped LFM wave are:Sample rate, pulse width, Pulse repetition frequency(PRF)Frequency step, number of steps, numsamples, numpulses.

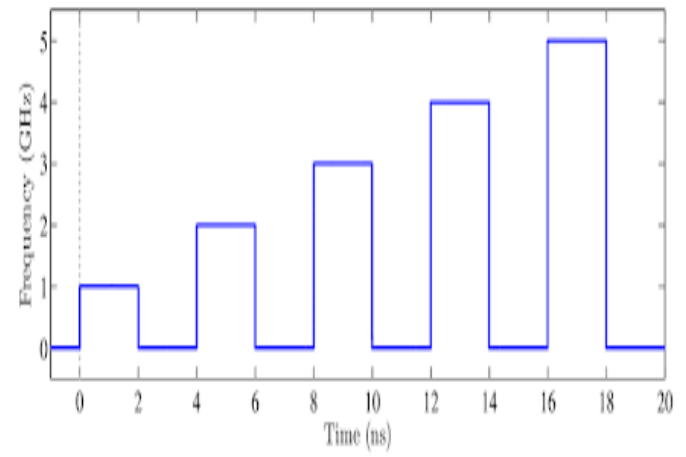

Figure 2 Stepped FM waveform (vi) Equation of Stepped FM

$Y=\cos \left(2 \quad\left(f t+(k / 2) t^{2}\right)+j \sin \left(2 \quad\left(f t+(k / 2) t^{2}\right)\right)\right.$

(4)

$$
\text { Where, } \quad \begin{aligned}
& k=\frac{f(i-1)-f(i)}{2 \times \tau} \ldots \ldots \ldots \ldots \text { (4) } \\
& \tau=\text { pulse width } \\
& f=\text { frequency } t=\text { time period }
\end{aligned}
$$

\section{SIMULATION RESULTS}

Simulation results of LFM,SF-CW with time domain and frequency domain is shown in Figure 5 to Figure 11.

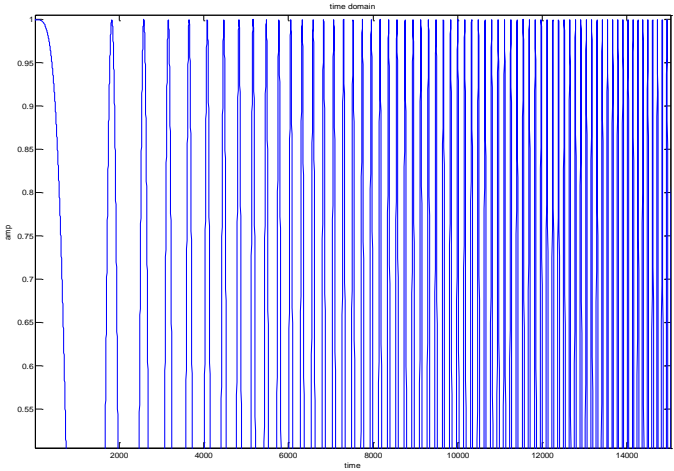

Figure 3 Time domain response of LFM

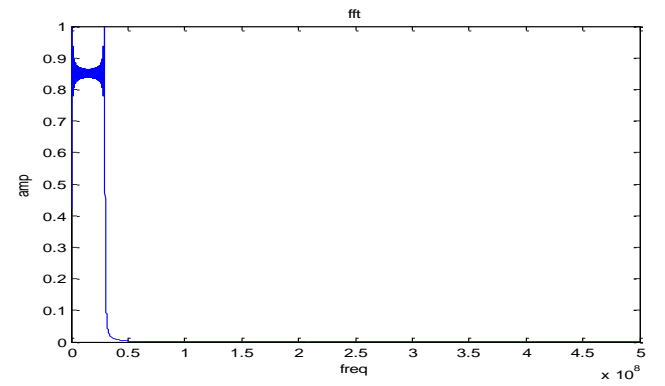

Figure 4 Frequency domain response LFM

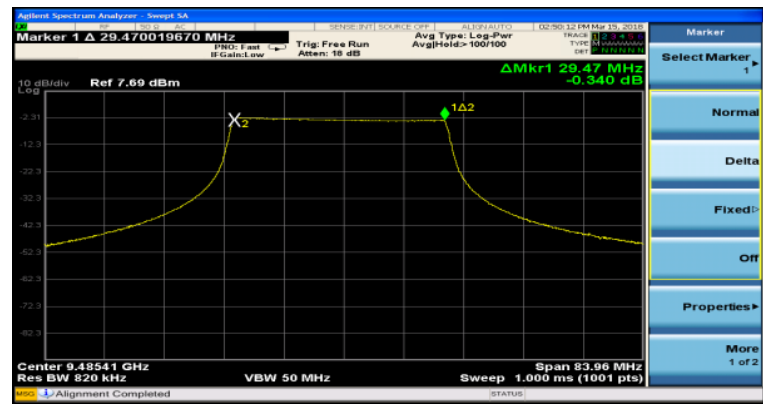

Figure 5 NI Suite representation of LFM

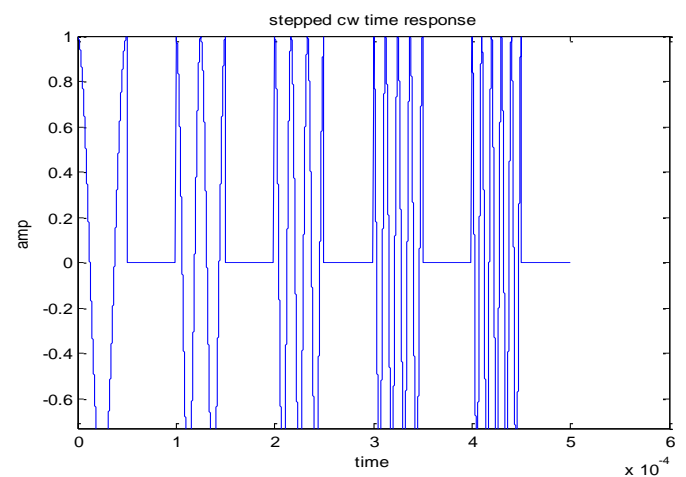

Figure 6 Time domain response of SF-CW

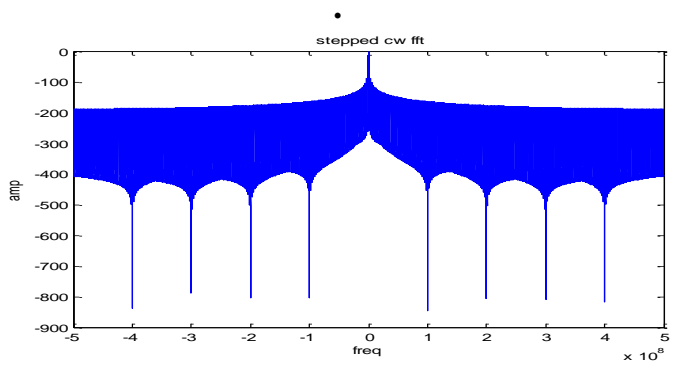

Figure 7 Frequency domain response SF-CW

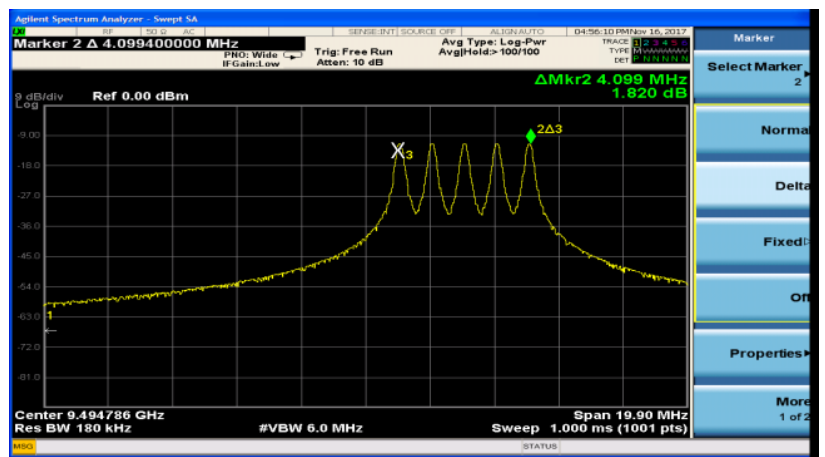

Figure 8 NI Suite representation of SF-CW

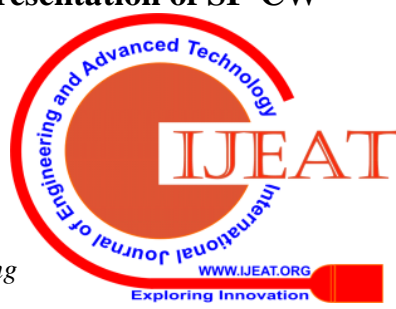




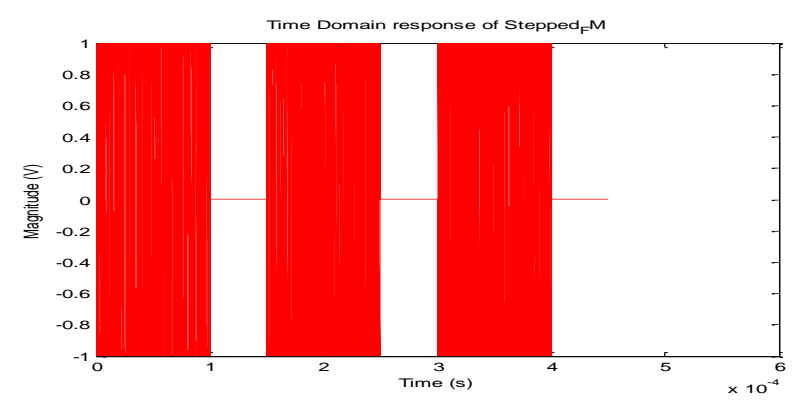

Figure 9 Time domain response of SF

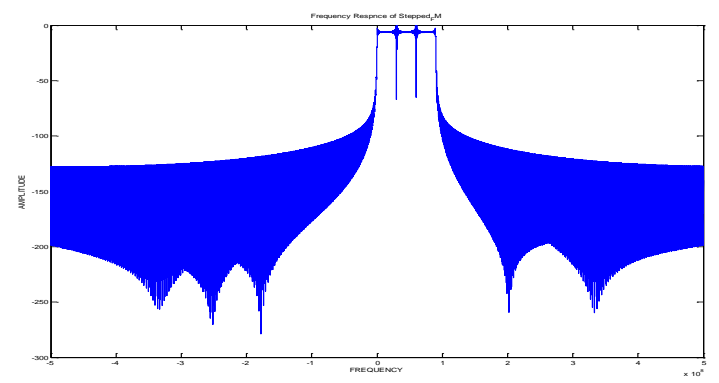

Figure 10 Frequency domain response of SF

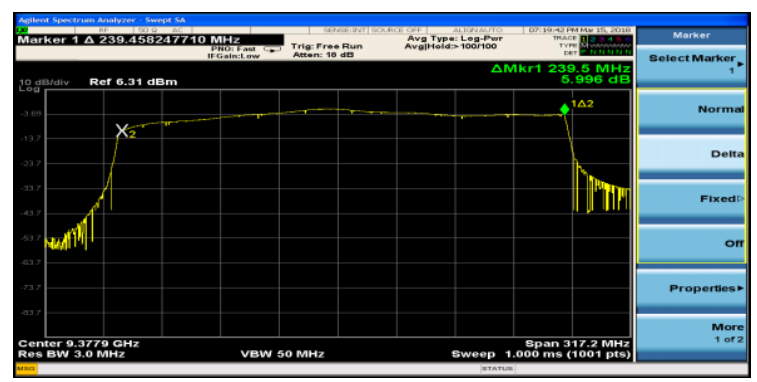

Figure 11 NI Suite representation of SF

\section{CONCLUSION}

The different techniques used for pulse compression are $\mathrm{L}$ inear Frequency Modulation Stepped Frequency Modulation and Step Frequency Continuous Wave in this report. By increasing the duration of a transmitted pulse, the LFM technique increases its energy and improves the capability of target detection. In contrast, increasing a pulse length increases the radar's range resolution. SFCW can compensate for the imperfections in the antenna and feed network of RF electronics and imperfections by postprocessing the data obtained. SFM will give Range Resolution and Frequency step length limits the non-ambiguous range. By using these techniques Better Range Resolution is achieved without High power transmitter and receiver. The results can be obtained by means of software MATLAB and NI Suite (SPECTRUM ANALYZER). This Presents that at the present time and in the future the complimentary codes can be successfully used in RADAR Systems.

\section{REFERENCES}

1. Vijay Ramya K, "A New Pulse Compression Technique for Polyphase Codes in Radar Signals", International Symposium on Devices MEMS, Intelligent Systems \& Communication (ISDMISC) 2011 Proceedings published by International Journal of Computer Applications (IJCA), Vol. 2, Issue 4, pp.15-17, 2011.

2. H. A. Said, A. "Design and Realization of Digital Radar Pulse Compression in Pulsed Radars Based on Linear Frequency Modulation (LFM) Waveforms Using FPGA", International
Conference on Advanced Information and Communication Technology for Education (ICAICTE), Published by Atlantis Press, pp.827-832, 2013.

3. Dodda. H.V.S. Rami Reddy, "Reduction of Side Lobes by Using Complementary Codes for Radar Application", IOSR Journal of Electronics and Communication Engineering, Volume 6, Issue 2, pp 27-30, 2013.

4. Anuja D. Sarate, "High Resolution Low Power Radar Pulse Compression Techniques", International Journal of Advanced Research in Electrical, Electronics and Instrumentation Engineering (IJAREEIE), Vol. 3, Issue 4, April 2014.

5. "Radar Basics for Pulse Compression" http://www.radartutorial.eu/08.transmitters/intrapulse\%20Modulation.e n.html,May10, 2016

6. M. I. Skolnik, "Introduction to radar," Radar Handbook, p. 1990, 1962.

7. [FanWang, Huotao Gao, Lin Zhou, Qingchen Zhou, Jie Shi, Yuxiang Sun, " Design and FPGA implementation of digital pulse compression for HF chirp radar based on modified orthogonal transformation", IEICE Electronics Express, Vol.8, P1736-1742, October-25-2011.

8. R.I Wijaya, S.N Ros, E.S Bagus, M Dadan, "FPGA based - Q chirp generator using first quadrant DDS compression for pulse compression radar", AIP conference proceedings, Vol 1755, Issue 1, 170005, 2016.

9. N U Azim, W Jun, "FPGA based hardware optimized implementation of signal processing system for LFM pulsed radar", Proceedings of the SPIE, Vol 10030, 2016.

10. Determination of PSL for FM and PM signals Jayshree Das, T Keerthi, I A Pasha,International Journal of Pure and Applied Mathematics Volume 118 No. 242018.

\section{AUTHOR'S FROFILE}

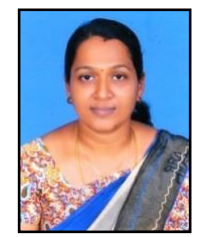

D.Tamilarasi, obtained her UG in ECE from Anna University,in the year 2005,PG degree in Power Electronics\&drives from Anna University in the year 2008 and PhD from Anna University in the year 2018.She is presently working as Associate Professor in MVJ college of Engineering,Bengaluru,India.She has 10 years of Teaching Experience. Her research areas are Power Electronics and Embedded System.

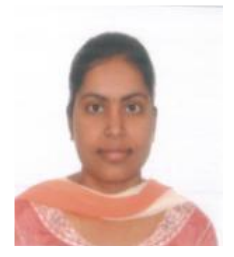

P.Pavithra, obtained her UG in Electronics and Instrumentation Engineering from Bharathiyar University,in the year 2003,PG degree in Applied Electronics from Anna University in the year 2005 .She is presently working as Assistant Professor in MVJ college of Engineering,Bengaluru,India.She has 5 years of Industrial Experience and 4 years of Teaching experience. Her research topics include IoT and automation, Computer Networks, Cloud Computing.

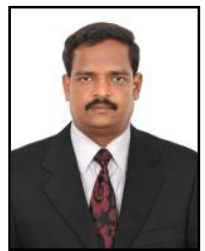

P.Ramesh, obtained his UG degree in Electrical Engineering from the Institution of Engineers (India), PG degree in Applied Electronics from Dr.MGR University, Chennai and $\mathrm{PhD}$ from Anna University, Chennai, in the year 2018. He is presently working as Professor and Head-Department Research Committee in CMR Institute of Technology, Bengaluru, India. He has 19 years of experience in teaching. His research topics include Power electronic applications in Renewable Energy Systems. 